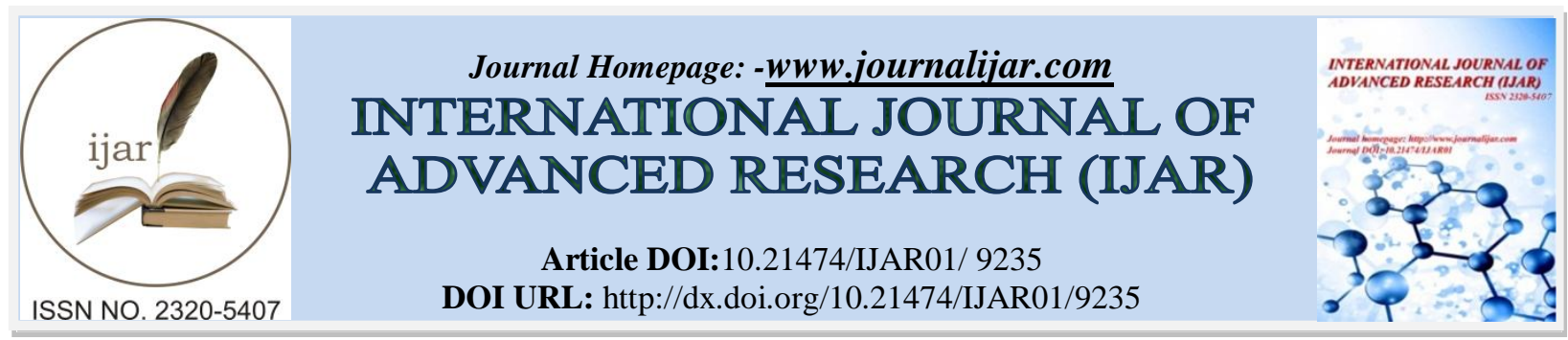

RESEARCH ARTICLE

\title{
RELATIONSHIP BETWEEN FOLLICULAR FLUID OXIDATIVE STRESS PARAMETER AND THE OUTCOME OF IVF-EMBRYO TRANSFER.
}

\section{Dr.Hina Kausar F.A.Ansari, Dr.D.V.Bhale and Dr.M.D.Hivre.}

Department of Biochemistry, MGM Medical College and Hospital, Aurangabad.

\section{Manuscript Info}

\section{Manuscript History}

Received: 08 April 2019

Final Accepted: 10 May 2019

Published: June 2019

Key Words: Female Infertility, Oxidative stress, In-vitro fertilization, Follicular fluid

\begin{abstract}
Oxidative energy production is unpreventably associated with the generation of reactive oxygen species. Germinal cells cannot be exempt, and may be exposed in the same manner as other cells to the devastating effects of oxygen metabolites.

Aim: To study the correlation of oxidative stress marker lipid hydroperoxide in preovulatory follicular fluid of infertile women undergoing in vitro fertilization and their pregnancy outcome.

Materials and methods: 60 patients undergoing in vitro fertilization were enrolled in the study. They were divided in two patient groups and compared in terms of follicular fluid lipid hydroperoxides and pregnancy outcome.
\end{abstract}

Result: Pregnant cycles were associated with significantly lower follicular fluid lipid peroxidation $(\mathrm{P}=0.001)$.

Conclusion: Our results demonstrate that elevated follicular fluid lipid peroxidation level may have a negative impact on In-vitro fertilization outcome. The findings support the idea that level of oxidative stress marker in follicular fluid may play an important role in fertility.

Copy Right, IJAR, 2019,. All rights reserved.

\section{Introduction:-}

Infertility is defined as inability of a couple to conceive naturally after one year of regular unprotected sexual intercourse. It remains a major gynecological problem, affecting $13 \%$ to $15 \%$ of couples worldwide as well as healthcare services and social environment. The prevalence varies widely, being less in developed countries and more in developing countries where limited resources for investigation and treatment are available. ${ }^{1}$ Infertility has been increased over the last thirty years, majorly in urban population due to social phenomena, such as the tendency for marriage at a later age and child bearing, increasing use of contraception specially Intrauterine device and liberalized abortion. ${ }^{2}$

In-vitro fertilization (IVF) a popular assisted reproduction technique (ART), is widely accepted procedure for the treatment of infertility. But unfortunately, the success rate of this technique is only $30-40 \%$. Therefore the assessment of oocyte quality in human in vitro fertilization (IVF) is getting increasing attention from embryologists. ${ }^{3}$ It is reasonable to think that some biochemical characteristics of the follicular fluid surrounding the oocyte may play a critical role in determining oocyte quality and the subsequent potential to achieve fertilization and

Corresponding Author:- Dr.Hina Kausar F.A.Ansari. 
embryo development. ${ }^{4}$ There are lot of research work done which suggests that excessive amounts of oxidative stress in follicular fluid may play a significant role in causing infertility. ${ }^{5}$

Oxidative energy production is unpreventably associated with the generation of reactive oxygen species. Germinal cells cannot be exempt, and may be exposed in the same manner as other cells to the devastating effects of oxygen metabolites. Data associating preovulatory follicle hypo oxygenation with high frequencies of oocyte cytoplasmic defects, impaired cleavage, and abnormalities in chromosome segregation have been reported by study by Van Blerkom et al., 1997. However, the biochemical background of these events remains unclear. As reduced oxygen supply is reflected in the modification of numerous metabolic pathways, intensified peroxidation may be one of the involved processes. ${ }^{6}$ Therefore; it was of interest to measure the concentrations of oxidative stress markers, namely lipid hydroperoxides in follicular fluid of women attending in-vitro fertilization (IVF) programme. Furthermore, these values were correlated with the outcome of IVF: fertilization and pregnancy rates.

In a healthy body, ROS (reactive oxygen species) and antioxidants remain balanced. When the balance is disrupted towards an overabundance of ROS, oxidative stress (OS) occurs. OS influences the entire reproductive lifespan of a woman and even thereafter (i.e. menopause). ROS are a double-edged sword - they serve as key signal molecules in physiological processes but also have a role in pathological processes involving the female reproductive tract. ROS affect multiple physiological processes from oocyte maturation to fertilization, embryo development and pregnancy. Many studies suggest that OS modulates the age-related decline in fertility, hence the present study was done to find out the relation between role of oxidative stress and outcome of embryo transfer in IVF.

\section{Materials and methods:-}

The present study was carried out in Department of Biochemistry, M.G.M. Medical College and Hospital, Aurangabad, in collaboration with Center for Reproductive Medicine, Fertility and Stem cell. The study was approved by Institutional Ethical and Research Committee. The study was conducted from November 2012 to July 2014. 60 Infertile females between the age group of 22-42 years were included in this study after taking informed consent from them.

\section{Inclusion criteria:}

Infertile female producing oocytes between the age group of 22-42 years and having their normospermic male partner, according to WHO 2010 guidelines for semen analysis and those undergoing their first IVF treatment cycle were selected. ${ }^{7}$

\section{Exclusion criteria:}

Following patients were excluded from the study:

1. Female patients not producing follicles which include those having ovarian failure and poor ovarian reserve

2. Female patients with any systemic disease like hypothyroidism, hyperthyroidism, diabetes mellitus, hyperprolactinemia

3. Female patients whose partners have Sperm count $<15$ million $/ \mathrm{ml}$, Motility $<60 \%$ and Grade 4 motility (Grade A) $<33 \%$

\section{Procedure:}

Infertile female reporting for IVF embryo transfer, was stimulated with a gonadotropin releasing hormone $(\mathrm{GnRH})$ agonist from mid luteal phase onwards and when optimally down regulated, were stimulated with recombinant follicular stimulating hormone (FSH). Follicular size was monitored regularly by ultrasound scan. Subcutaneously Human Chorionic Gonadotropin (HCG) was administered when average diameter of the leading follicles reached at least $18 \mathrm{~mm}$. Follicles having $18-20 \mathrm{~mm}$ diameter on ultrasound were selected.

Transvaginal oocyte retrival was performed under ultrasound guidance, $36 \mathrm{hr}$ after hCG administration. Microscopic examination of follicular fluid was performed by embryologist, to see the maturity of oocyte. Mature oocytes have second polar body and clear cytoplasm; whereas immature oocytes have no polar body. Oocytes were separated and placed into media, whereas follicular fluids were collected into separate sterile tubes. Only uncontaminated follicular fluids were retained for further determinations. After that mature oocyte were inseminated with spermatozoa. Embryo quality was assessed before embryo transfer by double inverted microscope and a maximum of two grade A embryo were transferred to all patients approximately $48 \mathrm{hr}$ (4-cell stage) after insemination. 


\section{Follicular fluid processing:}

After collection, follicular fluid samples were centrifuged at $2000 \mathrm{rpm}$ for 10 minutes to remove cellular components and the clear supernatant transferred to sterile tubes and kept at $-80 \mathrm{c}$ for no longer then 1 week.

\section{Laboratory analysis:}

Aliquots of the follicular fluid (FF) were thawed at room temperature and follicular fluid lipid peroxidation were assessed for MDA estimation by Thiobarbituric acid method. ${ }^{8}$

The chemicals and reagents used for the procedure were of analytical grade.

Pregnancy tests were performed on day 14 post embryo transfer, using a commercial urinary kit (UPT kit). ${ }^{9}$ Women with positive urinary pregnancy test were classified into the successful pregnancy group, while the women with negative urinary pregnancy test were considered the unsuccessful pregnancy group.

\section{Statistical Analysis:}

Mean and standard deviation were worked out for estimating the levels of follicular fluid MDA, in patients of female infertility. In order to compare these parameters the ' $p$ ' values (probability values) were obtained. ' $p$ ' value less than 0.05 was considered as statistically significant. The data was analyzed using SPSS version 20.

\section{Observation and result:-}

A total number of 60 infertile females included in this study were divided into two groups i.e. Group I (pregnancy positive group) includes 27 females and Group II (pregnancy negative group) includes 33 females.

Mean age (mean \pm SD) of Group I and Group II patients were $29.96 \pm 4.55$ years and 32.66 \pm 4.88 years respectively which were statistically significant $(\mathrm{p}<0.05)$. The patients in group I were significantly younger than that of group II.

Table No. 1:-Mean age of Group I and Group II patients.

\begin{tabular}{|c|c|c|c|}
\hline Variable & Group I $(\mathbf{n}=\mathbf{2 7})$ & Group II $(\mathbf{n}=\mathbf{3 3})$ & $\boldsymbol{p}$-value \\
\hline $\begin{array}{c}\text { Age (Years) } \\
\text { (Mean } \pm \text { SD) }\end{array}$ & $29.96 \pm 4.55$ & $32.66 \pm 4.88$ & $0.032 *$ \\
\hline
\end{tabular}

$(p<0.05$, Statistically significant $)$

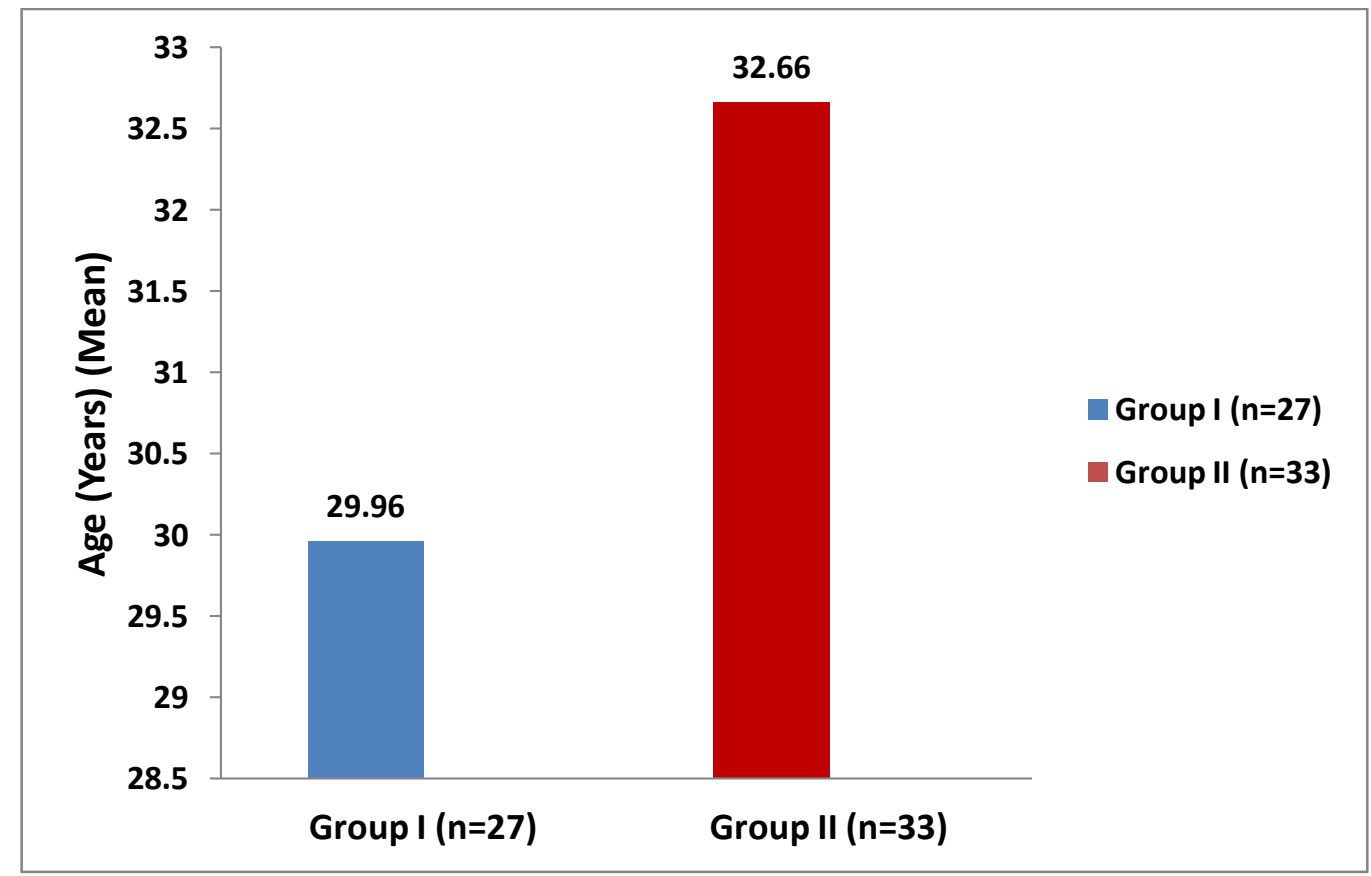

Bar Diagram No. 1:-Comparison of mean age between Group I and Group II patients 
Mean (mean \pm SD) follicular fluid MDA level in group I and group II patients were $0.90 \pm 00.44 \mathrm{nmol} / \mathrm{ml}$ and $1.35 \pm 0.43 \mathrm{nmol} / \mathrm{ml}$ respectively, as shown in Table No. 2. The statistical analysis by unpaired $t$-test showed that there was significant decrease in MDA level in group I compared to group II ( $p<0.001)$.

Table No. 2:-Mean follicular fluid MDA level in group I and group II patients.

\begin{tabular}{|l|l|l|l|}
\hline Variable & Group I $(\mathbf{n}=\mathbf{2 7})$ & Group II $(\mathbf{n}=\mathbf{3 3})$ & $\boldsymbol{p}$-value \\
\hline $\begin{array}{l}\text { MDA }(\mathrm{nmol} / \mathrm{ml}) \\
(\mathrm{Mean} \pm \mathrm{SD})\end{array}$ & $0.90 \pm 0.44$ & $1.35 \pm 0.43$ & $0.00^{*}$ \\
\hline
\end{tabular}

$(p<0.05$, Statistically significant $)$

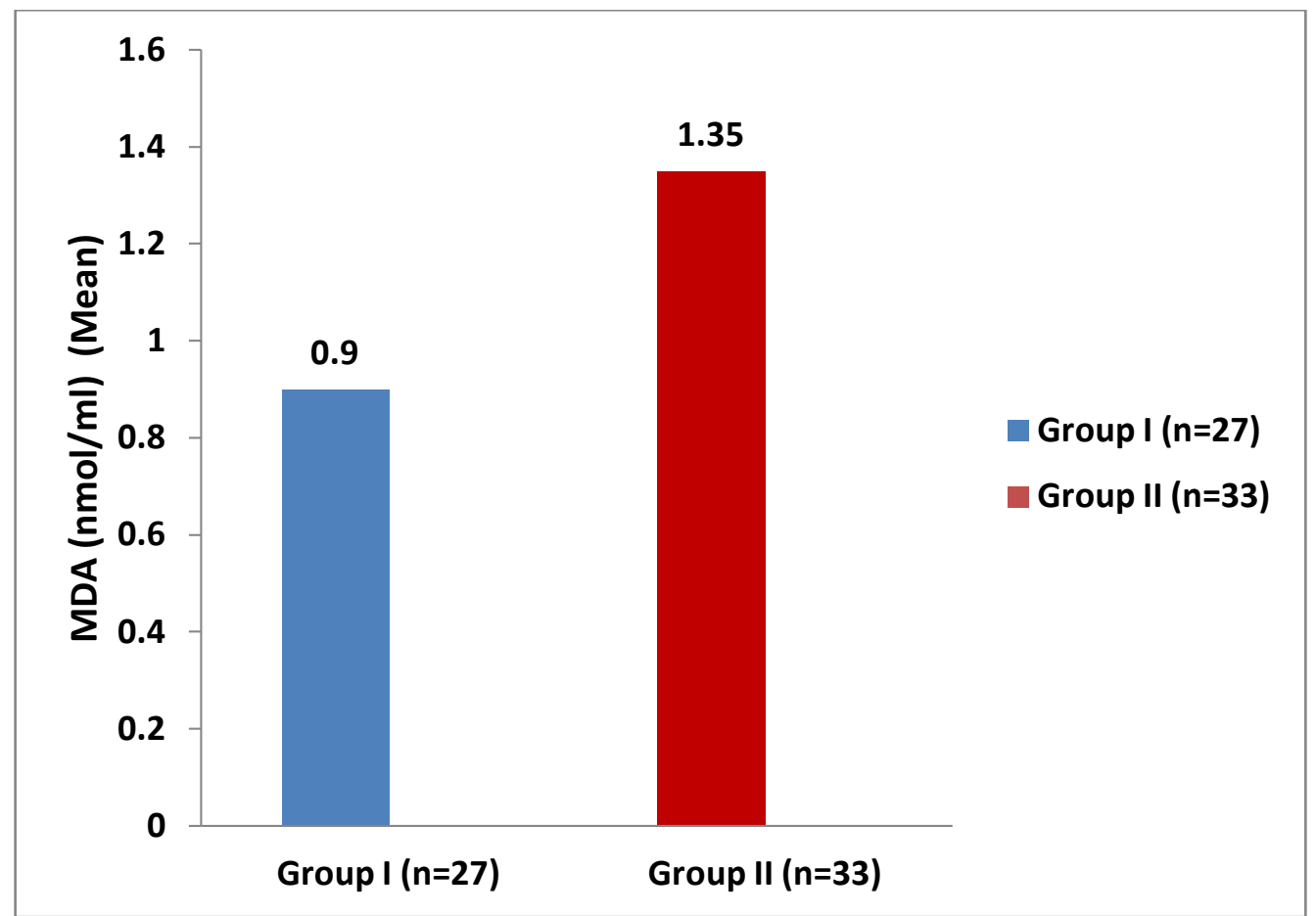

Bar Diagram No. 2:-Comparison of mean follicular fluid MDA level between group I and group II patients

\section{Discussion:-}

Infertility is a common problem experienced by many couples. Although numerous treatments are available for female infertility, but in some cases, the treatment is empirical in nature because the aetiology of infertility is not fully understood. Reactive oxygen species (ROS) plays an important role in normal reproduction and pathogenesis of infertility in females. Oxidative stress develops when there is an imbalance between the generation of ROS and the scavenging capacity of antioxidants in the reproductive tract. ${ }^{10}$ It affects both natural and assisted fertility. Treatments that reduce oxidative stress may help infertile women with diseases that are caused by this imbalance. Such strategies include identifying the source of excessive generation of ROS, treating the primary cause and invivo supplementation of antioxidants.

During IVF, the follicular fluid removed from the ovary has no therapeutic use and has become a "biological window" for understanding the environment of the oocyte in infertility. The oocyte is maintained with the components of the follicular fluid while maturing. Therefore, it is highly possible that some biochemical characteristics of the follicular fluid play a critical role on oocyte quality and the subsequent potential to achieve fertilization and embryo development. 
The biochemical composition of follicular fluid includes proteins, sugars, reactive oxygen species, antioxidants and hormones. Moreover, the oxidant-antioxidant state of follicular fluid and its effects on oocyte and IVF outcomes has been of great interest in recent years. ${ }^{4}$

Keeping this in view we investigated malondialdehyde (MDA), indicator of the oxidative status of the follicle, to predict the outcome of in vitro fertilization.

In our study we found mean age group of group I patients (preg +ve) were younger as compare to group II patients (preg-ve). ${ }^{11}$

According to our results, we have found that there was a statistically significant difference in follicular fluid MDA levels between pregnant and non-pregnant women. Mean (mean \pm SD) follicular fluid MDA level in group I and group II patients were $0.90 \pm 0.44 \mathrm{nmol} / \mathrm{ml}$ and $1.35 \pm 0.43 \mathrm{nmol} / \mathrm{ml}$ respectively. The statistical analysis showed that there was significant decrease in MDA level in group I compared to group II $(p<0.001)$. Our finding are in accordance with the study done by Das et al, ${ }^{12}$ they observed that there was a negative correlation between ROS levels and embryo quality. The study also suggested that concentration of oxidative stress markers in follicular fluid reflect the fertilization potential of oocyte. When the deleterious effects of free radicals on cell integrity are considered, it is expected that there has to be a negative correlation between peroxidation levels and IVF outcomes.

A study done by LIU Jing et al conclude that oxidative stress may induce apoptosis in granulosa cells and subsequently lower oocyte quality and lead to poor outcome of in vitro fertilization-embryo transfer (IVF-ET). In non-pregnant patients they observe significantly higher MDA level and higher incidence of apoptosis as compared to pregnant patients. A significant negative correlation was detected between MDA and fertilization rate. Our study showed similar findings. ${ }^{13}$

Yildirim B et $\mathrm{al}^{14}$ studied Lipid peroxidation in follicular fluid of women with polycystic ovary syndrome during assisted reproduction cycles. They found significant difference in follicular fluid MDA levels among the control and PCOS group.

Bedaiwy et $\mathrm{al}^{15}$ examined the role of ROS in embryo development in which high concentration of ROS were associated with lower pregnancy rates for IVF. This study showed that ROS affects not only fertilization rate and embryo development, but also the real outcome for a patient, in terms of the clinical pregnancy rate.

Study done by Yang et al (1998) ${ }^{16}$ showed that apoptosis was seen only in the fragmented embryos, indicating that high concentrations of hydrogen peroxide may cause embryo fragmentation. Therefore it suggests apoptosis is another process by which ROS affect embryo. In our study, group II subjects were having increase in MDA levels which causes damage to the embryo, hence pregnancy outcome was negative.

However, many studies have shown conflicting results in this respect as compare to our study. In a study by Appasamy et al, ${ }^{17}$ it was found that follicular fluid ROS levels had a positive correlation with the pregnancy rate in IVF patients. This observation made researchers think that a limited amount of oxidative stress may be essential for embryonic development since it is an indicator of metabolic activity. The results of a study by Pasqualotto et al ${ }^{18}$ showed parallel results, as pregnant women after IVF treatment had higher lipid peroxidation (LPO) products compared to non-pregnant women.

However, Oral et $\mathrm{al}^{19}$ found no significant relationship between follicular fluid malondialdehyde levels and fertilization rates.

\section{Conclusion:-}

It appears that the role of oxidative stress in relation to female reproduction needs further investigation and evaluation. In our study we found that decrease follicular fluid MDA level in group I women i.e pregnancy positive group while increase follicular fluid MDA level in group II women i.e. pregnancy negative group. Our results suggest that high levels of MDA in follicular fluid obtained from infertile women tend to decrease the fertilization potential of oocytes.

Future studies on large sample sizes of women with other causes of infertility undergoing IVF are required to highlight the critical role of oxidative stress markers and their optimum levels in female reproduction. This is 
expected to lead to improved ART success rate and infertility management. Additional studies are still needed to evaluate the use of antioxidants in ART setting to achieve higher live birth rate.

\section{Limitations:-}

More studies and sample size are required to measure biochemical parameters in follicular fluid, to assess and compare quality of oocytes and their effect on IVF outcome.

\section{References:-}

1. Kamel RM. Management of the infertile couple: an evidence based protocol. Reprod Bio Endocrinol 2010;21(8):1-8.

2. Mehrotra A, Katiyar DK, Agarwal A, Das V, Pant KK. Role of total antioxidant capacity and lipid peroxidation in fertile and infertile men. Biomedical Research 2013;24(3):347-52.

3. Das S, Chattopadhyay R, Ghosh S, Goswami SK, Chakraverty BN, Chaudhary K. Reactive oxygen species level in follicular fluid embryo quality marker in IVF? Hum Reprod Advance Access 2006 July;22:1-5.

4. Revelli A, Piane LD, Casano, Molinari E, Massobrio M, Rinaudo P. Follicular fluid content and oocyte quality: from single biochemical markers to metabolomics. Reprod Biol Endocrino 2009;7:5.

5. Ruder EH, Hartman TJ, Blumberg J, Goldman MB. Oxidative stress and antioxidants: exposure and impact on female fertility. Human reproduction Update 2008 Jul-Aug;14(4):345-57.

6. Jozwik M, Wolczynski S, Jozwik M, Szamatowicz M. Oxidative stress markers in preovulatory follicular fluid in humans. Mol Hum Reprod 1999;5:409-13.

7. World Health Organization WHO. Laboratory manual for the examination of human semen and semen-cervical mucus interaction, $4^{\text {th }}$ edi. Cambridge, UK7C Ambridge University Press, 1999 pp 4-23

8. Nourooz-Zadeh J, Tajaddini-Sarmadi J, McCarthy S, Betteridge DJ, Wolff SP. Elevated Levels of Authentic Plasma Hydroperoxides in NIDDM. Diabetes 1995;44:1054-58.

9. Salha O, Nugent D, Kaufmann S, Levett S, Jenner L, Sharma V. The relationship between follicular fluid aspirate volume and oocyte maturity in in-vitro fertilization cycles. Human Reprod 1998;13(7):1901-06.

10. Carbone MC, Taton C, Monache D, Marci R, Caserta D, Colonna RF. Amicarelli Antioxidant enzymatic defences in human follicular fluid: characterization and age-dependent changes. Molecular Human Reproduction 2003;9(11): 639-43.

11. Huang JYJ, Rosenwaks Z. In vitro fertilisation treatment and factors affecting success. Best Practice and Research Clinical Obstetrics and Gynaecology 2012;26:777-88.

12. Das S. Reactive oxygen species level in follicular fluid--embryo quality marker in IVF? Human Reproduction 2006 Jun 3;21(9):2403-7.

13. Jing LIU, Yanping LI. Effect of oxidative stress and apoptosis in granulosa cells on the outcome of IVF-ET. J Cent South Univ (Med Sci) 2010;35(9):990-94.

14. Yildirim B, Demir S, Temur I, Erdemir R, Kaleli B. Lipid peroxidation in follicular fluid of women with polycystic ovary syndrome during assisted reproduction cycles. J Reprod Med 2007 Aug; 52(8):722-6.

15. Bedaiwy MA, Elnashar SA, Goldberg JM, Sharma R, Mascha EJ, Arrigain S et al. Effect of follicular fluid oxidative stress parameters on intracytoplasmic sperm injection outcome. Gynecol Endocrinol 2012 Jan;28(1):51-5.

16. Yang HW, Hwang KJ, Kwon HC, Kim HS, Choi KW, Oh KS. Detection of reactive oxygen species and apoptosis in human fragmented embryos. Hum Reprod 1998;13:998-1002.

17. Appasamy M, Jauniaux E, Serhal P, Al-Qahtani A, Groome NP, Muttukrishna A. Evaluation of the relationship between follicular fluid oxidative stress, ovarian hormones and response to gonadotropin stimulation. Fertil Steril 2008;89:912-21.

18. Paszkowski T, Traub AI, Robinson SY, McMaster D: Selenium dependent glutathione peroxidase activity in human follicular fluid. Clin Chim Acta 1995;236:173-80.

19. Oral O, Kutlu T, Aksoy E, Fıçıcıoğlu C, Uslu H, Tuğrul S. The effects of oxidative stress on outcomes of assisted reproductive techniques. J Assist Reprod Genet 2006;23:81-5. 